\title{
Antecedents, Processes, and Outcomes of an Interdisciplinary, Conference/Collaboration: A comparative Study of Three Interdisciplinary Working Groups
}

\author{
Juliana L. Fuqua al, Anna Bargagliotti ${ }^{\mathrm{b}}$, Jeffrey A. Phillips ${ }^{\mathrm{c}}$, Dorothea Herreinerd, Laura \\ DaSilva $^{\mathrm{a}}$
}

${ }^{\mathrm{a} C a l i f o r n i a ~ S t a t e ~ P o l y t e c h n i c ~ U n i v e r s i t y, ~ P o m o n a, ~ U S A ; ~}{ }^{\mathrm{b}}$ Loyola Marymount University, USA

\begin{abstract}
There is a growing recognition of the need for interdisciplinarity in science, technology, engineering and mathematics (STEM) education. The purpose of the present study is to identify antecedents, processes, and outcomes of an interdisciplinary, collaborative conference and ongoing collaboration. The Breaking Boundaries in STEM education conference was developed with multiple goals, including fostering collaborative interdisciplinary scientific writing for publication among teacher-scholars who participated in one of three interdisciplinary working groups. One hundred teacher-scholars with interest in STEM education participated in the conference. A comparative study of three working groups from the conference was conducted using a triangulation of qualitative and quantitative methods. Surveys and behavioral observations were completed at the conference, and phone interviews with attendees were conducted 3-4 months later. Groups varied in their readiness to collaborate. Several themes emerged that might explain why one group was highly productive, one group was moderately productive, and one group was not productive at completing publications after the conference. Groups with a narrower disciplinary span, stronger leadership presence, a paper champion, motivated leader, and a leader with a strong recent history of publishing on the topic, were more ready to collaborate, and they experienced faster, smoother completion of publications. Further research and more passage of time, such as a few years, is needed to determine the quantity, quality, span of disciplinarity, novelty, and generativity of the publications over time. The generalizability of these themes to other interdisciplinary collaborative studies is briefly discussed.
\end{abstract}

Keywords: Problem solving, interdisciplinary activities, problem choice, disciplinary tasks

\section{Introduction}

There is growing societal recognition of the need for interdisciplinary research in STEM education. For example, in order to enhance contributions of discipline-based education research to the understanding of undergraduate science and engineering education, the National Research Council recommended that interdisciplinary studies of cross-cutting concepts are needed (National Research Council, 2012). In recognition of the potential benefits of interdisciplinary efforts (including better solutions to societal problems and challenges), small-scale grants and very large initiatives have been established to promote interdisciplinary collaborative intellectual innovations (e.g., publications). Over the past two decades, large-scale efforts have grown with increasing investment by government and private foundations in initiatives. The National Cancer Institute's \$70-million initiative established Transdisciplinary Tobacco Use Research Centers at seven universities in 1999

${ }^{1}$ Department of Psychology \& Sociology, California State Polytechnic University - Pomona, California, USA, E-mail: jfuqua@cpp.edu

Fuqua, J. L., Bargagliotti, A., Phillips, J. A., Herreiner, D., \& DaSilva, L. (2018). Antecedents, Processes, and Outcomes of an Interdisciplinary, Conference/ Collaboration: A comparative Study of Three Interdisciplinary Working Groups. Journal of Research in STEM Education, 4(1), $101-116$. 
(National Cancer Institute, 1999). Center applicants had to incorporate a transdisciplinary model (Rosenfield, 1992) indicating how they would strive to achieve transdisciplinarity. The Breaking Boundaries conference exemplifies a small-scale effort, and the conference is evaluated in this paper.

\section{Transdisciplinary collaboration}

Transdisciplinarity is an ideal goal of many interdisciplinary collaborative efforts. Transdisciplinarity denotes the strongest form of interdisciplinarity with the highest level of interaction among collaborators and can be defined as a process in which individuals work jointly using a shared conceptual framework that draws together discipline-specific theories, concepts, and approaches to address a common problem (Rosenfield, 1992). Transdisciplinary collaborative research has been described as collaboration among scholars representing two or more disciplines, with the products of that collaboration reflecting an integration of conceptual and/ or methodological perspectives drawn from two or more fields. In the present paper, we will focus broadly on interdisciplinarity rather than transdisciplinarity specifically. For more information about interdisciplinarity and the factors that influence the success of transdisciplinary scientific collaboration, please see what has become a vast literature, which includes empirical articles and books by Daniel Stokols, Juliana Fuqua, and Julie Thompson Klein (e.g., Stokols, 2008; Fuqua, Stokols, Gress, Harvey \& Phillips, 2004; Klein, 1996).

In the present paper, we discuss interview themes from an evaluation of the Breaking Boundaries in STEM-Ed conference/collaboration, a new regional, interdisciplinary conference/collaboration. We identify the antecedents, processes and outcomes of the conference/collaboration that can be generalized to other interdisciplinary scholarly collaborative endeavors. Social psychology, organizational psychology, preventive medicine and health promotion studies, and the science of team science provide our lens through which we view findings.

\section{Background: About the conference}

An interdisciplinary conference/collaboration ${ }^{1}$, Breaking Boundaries in STEM Education, was held on April 7, 2017, to discuss developments and foster opportunities in undergraduate STEM-Ed locally in Southern California. Building on the goal of increasing the number and diversity of students in STEM pathways as well as improving those students' skill sets, the proposed conference was organized around three timely and important themes in STEM education: (1) equity, (2) problem-solving, and (3) computational thinking.

These themes served as the guiding framework to organize participants. All three themes are pressing issues in STEM education that can be studied through discipline-based education research (DBER) and scholarship of teaching and learning (SoTL) research. For each theme, there was a contributed paper session, workshop, and working group. The goal was to engage in a deep discussion of how DBER and SoTL research can help shed light on commonalities as well as how challenges can be approached across disciplines for each theme. The organization of the conference was led by CREATE-STEM (or Collaborative Research on Evaluating, Advancing, and Transforming Education in STEM, http://www.create-stem.org) and the Center for Teaching Excellence (http://academics.lmu.edu/cte). Both Loyola Marymount University organizations have wide visibility in the Southern California area, and the individuals who have run these organizations helped organize the Breaking Boundaries conference.

The Breaking Boundaries conference had several aims, one of which was for attendees to share ideas and collaborate to write a manuscript for publication. To combat the problem that STEM-Ed scholars have no (or almost no) venues to publish cross-disciplinary work, the organizers sought a new journal, Journal of Research in STEM Education (or J-STEM) and arranged for a special issue to be written by conference working groups. The three working groups at the conference were expected to collaborate on the conference day and

${ }^{1}$ This conference was designed to help facilitate an ongoing, local collaboration or network among STEM-Education researchers and interested others. Organizers debated whether they were mainly running a conference or an ongoing collaboration. Organizers could not find another word that accurately captured the collaborative nature of the conference and the ongoing collaboration. For the sake of simplicity, "conference" is the term used in this paper to refer to both the conference and ongoing collaboration. 
after the conference, before submitting manuscripts to the journal. The submission ideas were expected to emerge and be influenced by the conference working group.

There are many other ways to define the success of a conference/collaboration besides publications. In fact, the aims of the Breaking Boundaries conference/collaboration were to help STEM education scholars, researchers, and teachers do the following:

- learn about new scholarly work on three themes (problem-solving skills, computational skills, and equity);

- be inspired to gather more information about the themes (problem-solving skills, computational skills, and equity);

- be inspired to change teaching practices;

- be inspired to pursue new research ideas or pathways;

- find potential future collaborators on STEM-Ed relevant research;

- cultivate future collaborators which would lead to new outcomes, such as "seeds" (or ideas and intellectual products (including a publication manuscript, grant proposals, or conference presentations).

Evaluating the full impact of the Breaking Boundaries conference is beyond the scope of the current paper.

The Breaking Boundaries conference was structured to include traditional conference sessions of plenary talks, conference presentations, and a poster session as well as more innovative workshops and three working groups organized by themes (problem-solving, computational thinking, and equity). Attendees selfselected into working groups based on their interest. In an effort to enhance confidentiality, the working groups will be referred to, below, as Groups A, B, and C.

In this paper, we use comparative analysis to analyze antecedents and processes that may have influenced each group's productivity, defined as completing interdisciplinary, collaborative manuscripts.

\section{Research questions}

What antecedents and processes might impact interdisciplinary, collaborative efforts? Specifically, what factors might explain why three interdisciplinary working groups differed in their readiness to generate publication manuscripts based on group discussions?

\section{Methods}

Approximately 100 people registered for the one-day Breaking Boundaries conference, held in April 2017. An estimated $90 \%$ of registrants came to the conference and participated. An official count was not taken.

Before the conference, three conference organizers (the principal investigator and co-principal investigators of the grant) led the development and oversight of the conference. They decided to divide participants into three working groups. Conference organizers recruited two or three group leaders to develop and run each working group (Groups A, B, and C). Multiple meetings and conversations were held between conference organizers and group leaders for several months prior to the conference.

Behavioral observations, surveys, and interviews of conference participants serve as the data source for the present paper. At the conference, behavioral observations were made informally at multiple times. In addition, a brief evaluation form which was written by a conference organizer was distributed after the afternoon working group session (as well as other conference sessions). The form instructed attendees to use a scale of 1 (low) to 5 (high) to rate the session's content, presentation, opportunities for engagement, interaction 
with others, and interdisciplinary exchange.

A few months after the conference, interviews were conducted with 10 conference participants in July and August 2017 by phone (or by email with one participant). Much of the present report is based on these interviews. Interviews ranged from 20 minutes to over three hours in length, and occasionally an interview was conducted over a few days. Before interviews began, conference organizers decided to select participants who represented diverse backgrounds. Diversity was not defined in terms of ethnicity or race, but in terms of academic discipline (e.g., mathematics, physics, computer science), their rank (e.g., assistant professor), centrality to conference leadership (conference organizer, group leader, non-leader), and knowledge of STEMEd research (minimal vs. extensive).

Interview questions were developed to assess the factors that influence the collaborative processes and outcomes before, during, and after the conference. For example, respondents were asked to describe the extent to which they have established new intellectual linkages with other conference attendees and actually began intellectual linkages as a result of the conference (such as working on a conference publication).

\section{Results and Discussion}

Factors that influence collaborative readiness to develop interdisciplinary products (i.e., publications)

Four months after the conference, it was clear that the working groups differed greatly in their readiness to complete a collaborative manuscript for publication fully or partially based on group discussions. This difference was also clear during the conference. Group A showed the most collaborative readiness, and they published collaborative interdisciplinary papers. Group B has made progress on but has not completed any papers by the time the present report was written. Group $\mathrm{C}$ showed the least readiness and has not worked on drafts of publication manuscripts. Next, we present several antecedents and processes that may explain why Group A was the most ready to collaborate. Many of the antecedents, processes, and outcomes are listed in Table A. Some factors are not listed due to lack of complete data from all three groups.

What is a narrow disciplinary span? A narrow disciplinary span characterized Groups A and B more than Group C. What is a narrow disciplinary span? Interdisciplinary collaborative endeavors can be characterized as narrow or broad in disciplinary span of the collaborators. Previous scholars have provided a typology of interdisciplinary research, and they suggest that disciplines range from narrow to wide in span. Narrow interdisciplinarity involves: (a) interactions between disciplines with the same paradigms, same methods, and disciplinarity outputs that can be easily integrated; (b) few disciplines involved (which simplifies communication), (c) representatives of disciplines are in the same organization, and (d) representatives from disciplines that share the same disciplinary culture (Van Dusselddorp \& Wigboldus, 1994). We refer to "narrow disciplinary span" as a small range of 2 or more disciplines which are similar (e.g., rely on similar methods of analyzing phenomenon and share similar world views). Mathematics and physics are disciplines that are similar and exemplify a narrow disciplinary span whereas sociology and biology would constitute a large disciplinary span.

It can be difficult to categorize interdisciplinary span when scholars are from different disciplines but share similar fields of expertise. A collaboration that is categorized only by discipline may appear to have a large span, but might actually have a narrow span. Biologists who study biostatistics and mathematicians who study statistics may have much in common. Also, psychologists who study and teach neuroscience and the functioning of the prefrontal cortex of the brain have much in common with neuroscientists. Psychologists who study team effectiveness have much in common with certain business and management professors. 
Table A.

Comparative table of antecedent conditions, processes, and outcomes for the three working groups

\begin{tabular}{|c|c|c|c|}
\hline & Group A & Group B & Group C \\
\hline Overall readiness to publish & High & Medium & Low \\
\hline \multicolumn{4}{|l|}{ Antecedents and processes } \\
\hline Disciplinary span: narrow vs. wide & Narrow & Moderate & Wide \\
\hline Group has a paper champion & Yes & Somewhat & No \\
\hline Group has a shared history & Yes & Yes & $\begin{array}{l}\text { Not clear from inter- } \\
\text { views }\end{array}$ \\
\hline $\begin{array}{l}\text { Physical environment: collaborator offices are } \\
\text { spatially proximal }\end{array}$ & $\begin{array}{l}\text { At least two members' } \\
\text { offices are proximal }\end{array}$ & $\begin{array}{l}\text { Not mentioned in } \\
\text { interviews }\end{array}$ & $\begin{array}{l}\text { Not mentioned in } \\
\text { interviews }\end{array}$ \\
\hline $\begin{array}{l}\text { Group leaders have published recently on } \\
\text { topic }\end{array}$ & Yes & Yes & No \\
\hline \multicolumn{4}{|l|}{$\begin{array}{l}\text { (Note: Group leaders had great expertise } \\
\text { in the topics, but some had not published } \\
\text { recently on the topic.) }\end{array}$} \\
\hline $\begin{array}{l}\text { Group leaders' conference organizing expe- } \\
\text { rience }\end{array}$ & Strong & Strong & $\begin{array}{l}\text { Not consistently } \\
\text { strong across leaders }\end{array}$ \\
\hline Group size & Rather small & Medium & Rather large \\
\hline $\begin{array}{l}\text { Physical environment: meeting space charac- } \\
\text { teristics }\end{array}$ & $\begin{array}{l}\text { Small room with one } \\
\text { table }\end{array}$ & $\begin{array}{l}\text { Medium-size class- } \\
\text { room }\end{array}$ & Large classroom \\
\hline \multirow[t]{2}{*}{ Leadership: Oversight by organizers } & High & High & Low \\
\hline & $\begin{array}{l}\text { Conference organizers } \\
\text { were at the working } \\
\text { group }\end{array}$ & $\begin{array}{l}\text { Conference organizer } \\
\text { was at the working } \\
\text { group }\end{array}$ & $\begin{array}{l}\text { No conference orga- } \\
\text { nizer was at the group }\end{array}$ \\
\hline \multicolumn{4}{|l|}{ Outcomes } \\
\hline Publication progress as of 4 months later & $\begin{array}{l}\text { Major progress. Publi- } \\
\text { cations submitted }\end{array}$ & $\begin{array}{l}\text { Moderate progress. } \\
\text { Publications in } \\
\text { progress. }\end{array}$ & $\begin{array}{l}\text { Minimal } \\
\text { Progress. }\end{array}$ \\
\hline $\begin{array}{l}\text { Potential for publishing transdisciplinary, } \\
\text { collaborative papers }\end{array}$ & $\begin{array}{l}\text { Unclear - analysis of } \\
\text { papers needed }\end{array}$ & $\begin{array}{l}\text { Unclear - analysis of } \\
\text { papers needed }\end{array}$ & Weak \\
\hline
\end{tabular}

Scholars have noted that interdisciplinary collaborations can be characterized as having "horizontal" integration or "vertical" integration between disciplines (Stokols, Fuqua, Gress et al. 2003). Horizontal integration occurs between similar disciplines that share similar levels of analysis (e.g., physics and mathematics). Vertical integration between disciplines involves linkages between disciplinary concepts that are much more distant in their levels of analysis micro levels of analysis is conducted by geneticists and macro level of analysis is conducted by sociologists).

How might a narrow disciplinary span impact groups? Groups with a narrow disciplinary span are less diverse than those with a wide disciplinary span. Previous social and organizational psychological research supports the notion that group diversity has an impact on group processes and performance. ${ }^{2}$

\footnotetext{
${ }^{1}$ Source: Langford (in press) and personal communication with Dr. Sara Langford, author of the encyclopedia entry "Diversity and teams".
} 
Diversity has been defined in multiple ways. Scholars have indicated that diversity is often defined and studied in terms of demographics (or similarly "bio-demographics", "surface level" variables, or "relationsoriented" variables) such as age, gender, ethnicity, and race. Another definition of diversity is more central to the core of an individual (or "deeper" including cognitive ability, personality traits, values, beliefs, and attitudes, Harrison, Price, Gavin, \& Florey, 2002). Compared to diversity defined as demographics, "deeper-level" diversity has been found to be have a stronger impact on team dynamics and team process outcomes (Woehr, Arciniega, \& Poling, 2013) as well as performance (Vodosek, 2007). In a study of team effectiveness (Woehr, Arciniega, \& Poling, 2013), a 40-item scale was used to assess individuals' values, including whether an individual values being creative and thinking up new ideas. Deep-level similarity resulted in more team cohesion, less conflict, and greater efficacy. In another study, diversity was defined as group members' dissimilarity in horizontal and vertical individualism and collectivism (or cultural diversity, Vodosek, 2007). Group diversity was found to be associated with satisfaction with the group and perceived performance of the group. Group similarity was adversely related to three types of intragroup conflict types (relationship, process, and task conflict), which were associated with unfavorable group outcomes (e.g., satisfaction with the group and productivity).

There is some consensus among psychologists that diversity (particularly task-oriented diversity) can impede group cohesion and enhance creativity, but the impact on task performance is not clear (Langford, in press). More group similarity (defined in terms of values and attitudes) may lead to less conflict, more cohesion, more satisfaction, and more rapid and immediate productivity. Alternatively, more group diversity is associated with more conflict, less cohesion, less satisfaction, and less rapid, immediate productivity (Vodosek, 2007; Woehr, Arciniega, \& Poling, 2013). Cross-functional teams (which means diversity is defined in terms of subject-matter knowledge) increases conflict while completing a task, but also improves creativity and performance in general (e.g., increased effectiveness and efficiency; Langford, in press).

According to empirical studies of research centers, a narrow disciplinary span can facilitate a quicker, more smooth-running collaboration that can lead to more likely completion of tasks such as the development of intellectual outcomes (e.g., conference presentations and publications), as illustrated in a study of two transdisciplinary scientific collaborative research centers. A wide disciplinary span can lead to collaborators having communication difficulties including speaking different disciplinary 'languages' and having different world views, which can lead to conflict and challenges with productivity. A narrow disciplinary span might lead to more rapidly developed intellectual outcomes yet may not necessarily lead to a very novel product that generates many other new ideas. A wide disciplinary span can lead to more novel outcomes that could potentially have an even greater impact (Fuqua, 2002; Fuqua, et al. 2004). Entire disciplines and fields that are novel and highly generative of new ideas come from wide disciplinary spans. For example, psychoneuroimmunology emerged from two very different disciplines and changed psychologists' and immunologists' understanding of stress.

Disciplinary span of groups A, B, and C. Groups with a narrower disciplinary span were more likely to publish manuscripts. Behavioral observations of Group A suggest that the members shared similar disciplines (e.g., mathematics and physics), Group B shared at least four similar disciplines, and Group C had many very different disciplines (including physics, mathematics, and psychology). (A full analysis of the disciplines of each group are not available due to lack of data. Future research could include such analysis, including an analysis of the extent to which vertical and horizontal integration between disciplines was attempted and occurred).

Group A members were able to have some shared understanding of their topic and of the concepts they were discussing. They shared enough language and concepts to discuss their topic well from their different disciplinary perspectives. Interviewees commented that they enjoyed learning from one another about how their topic was defined. They were able to identify similarities and differences in their disciplinary approaches. Two respondents noted that they gained new ideas from these discussions and began working on a publication. They were from the same department (and in the same discipline), and they had not previously collaborated. 
Not everyone who entered Group A stayed. The disciplinary span was perceived as too narrow for some individuals. One social science professor entered the group and commented that his/her disciplinary background was too different from the others. The professor felt intellectually excluded and described the experience:

"I walked into the working group session a little late and saw a group of people looking at handouts with many formulas on them and talking about [the group topic]. I found it to be a little intimidating, confusing, and off-putting because I could not understand the written symbols on the handouts nor could I understand what the group was talking about. My discipline does not require students to do such work. The topic is not a major part of my discipline, and we discuss it differently... much more macro. We rarely assign homework that requires those skills, and rarely are numbers needed. The group members [Group A members] seemed to share disciplinary language and concepts in a way that I could not. Although someone who was leading the group was very nice, enthusiastic, and encouraged me enthusiastically to stay, I felt intellectually isolated and unable to contribute or benefit from these conversations. I decided to leave and find a new group."

Although several disciplines were represented (biology, mathematics, statistics, computer science), the group may have shared similar perspectives. The similarity in perspective may have helped the group efficiently collaborate. In the group, two similar disciplinary perspectives dominated, according to an interviewee. Multiple professors had experience thinking about and working on the same topic but from different perspectives (and some of them had worked together on publications before).

Group B attendees were not sure of the interdisciplinary span of their group, but they indicated that the group shared at least four similar disciplines, including mathematics economics, and statistics. Their answers suggest that the span was moderately wide, but more data is needed to determine the span of Group B. Group C was represented by a wide span of disciplines. Social scientists, including psychologists, were together with physicists, chemists, and others. The topic did not require knowledge of numerical formulas, and thus attracted individuals representing multiple disciplines. There were no handouts with formulas. One social science scholar commented,

"As I looked around the room at the attendees, the handouts, and the presentation slides, it appeared that we had a wide range of disciplines represented. I felt that scholars from many disciplines would be able to understand the concepts we were discussing. Although we all probably understood the presentations and discussions, we did not figure out how to bridge ideas or start a manuscript together."

In addition, one interviewee noted that the topic was not closely related to what STEM educators are required to know in order to do their jobs. The topic is traditionally considered a social science topic, and it is not a concept that is taught to most STEM students on a regular basis.

One paper "champion" (or motivated paper leader) A commonly endorsed viewpoint was, "A paper needs a champion, meaning at least one person who is highly motivated to take on a collaborative project such as leading the writing of a manuscript for publication. If no one is highly motivated, it won't happen."

Group A and Group B but not Group C had paper champions. In Group A, one person took initiative and acted as a paper champion. This person was not assigned to lead the group but might have been motivated to take on the role due to a strong interest, a strong background in the topic, and a job rank (not full professor) in which publications are required for future promotion. Group B had a paper champion who was assigned to help lead the group. Group C had members assigned to lead the group, but they did not have a paper champion.

What makes a paper champion? What makes a paper champion willing to collaborate across disciplinary bounds? Many factors may contribute to a person becoming a paper champion. A few are listed in the present paper (e.g., past history of publishing on the topic). Daniel Stokols and colleagues have argued that a "transdisciplinary ethic" (Stokols, 1999; Stokols, Fuqua, et al., 2002 may be essential among interdisciplinary 
scientific authors working to achieve intellectual outcomes that are novel and merge disciplinary concepts. Transdisciplinarity refers to the strongest form of interdisciplinarity (Rosenfield, 1999; Stokols, 1999). A transdisciplinary ethic is characterized by factors such as openness to collaborate. Interview responses suggest that the champions of the papers in Group A and B showed characteristics of a transdisciplinary ethic.

In the future, a 'transdisciplinary ethic' scale could be given to potential collaborators and leaders to predict who is the most likely to be open to collaborate. No such scale was used for the Breaking Boundaries conference.

Shared working history and social cohesion, social capital, and informality of relationships. Even though a person may want to champion a paper (and be willing to spend sufficient time writing it), other factors matter. A shared working history and/or social cohesion, social capital, and informality of relationships might contribute to smooth working relationships with collaborators, which may foster more rapid productivity (Fuqua et al., 2004).

There was insufficient data to fully analyze these factors, but some evidence suggests a pattern. In Group A, at least a few members had built up social capital, informal relationships, and a shared working history--working together in some capacity for multiple years. For example, of couple of people collaborated for multiple years as officers in a discipline-specific organization. In Group A, some members shared working history in organizations. In Group B, at least two members had written a manuscript before and continued to work together to write a manuscript for the group. In Group C, the working history of members was less clear, according to interviews. It seemed as if the Group C leaders did not have a strong history of working together on projects, according to one respondent.

Having such helpful processes helped group A and B members. "It was easy to contact my writing partner and ask a few questions. I trusted his response. We worked well informally," said one respondent. "I didn't have to write a formal email, wait for a response, etc."

Spatial proximity of collaborators. Spatial proximity of collaborators may also be associated with more time spent in communication, social cohesion, and collaborative productivity (Festinger, Schachter, \& Back, 1950; Fuqua et al., 2004; Zhan, 1991). Some productive collaborators mentioned that they share a hallway and department with their collaborator (a paper champion). Their responses suggested that they appreciated working down the hall from each other. Environmental psychologists have suggested that sharing a physical space such as a hallway at work where you pass by one another regularly can lead people to get to know one another and build up more trust, informal relationships, and friendliness. The actual physical distance between individuals "as the crow flies" matters less than the shared spatial distance (or functional distance). Interdisciplinary scientific collaborators who share hallways in the same building were found to be more likely to develop intellectual innovations such as publications (Fuqua, 2002).

Sharing space and time together outside of one's work office can also be helpful. (What is the point of scientific conferences if they don't help transmit ideas that can lead to improved intellectual and educational innovations?) One interviewee mentioned that although his/her office is in the same building as another conference attendee, the off-campus conference location was particularly helpful to them. With some recognition that collaboration may be best fostered off campus (even when one shares a building on campus), one respondent happily explained, "I have been thinking about working more with [name deleted]. We have done a little prior collaborative work together. I guess we needed a conference more than 30 miles from campus to get us to together in the same space with time to talk about our mutual research interests, even though our offices are relatively close." The respondent added, "We don't normally go to the same discipline-specific research conferences, so I appreciated the opportunity to go to a local conference [in the same place] which did not require a lot of travel and to be at the same conference with a more general theme of STEM that works for both of us. 
Past history and motivation to publish on the topic. A strong recent history of publishing may reflect an individual's current motivation to publish. Multiple recent publications on the topic were evident in Group A and B leaders' publication history (according to online faculty pages). In fact, the topic of the group was in the title of several past recent publications of Group A and Group B paper champions.

All of the group leaders had strong, positive reputations as experts with exceptional knowledge and strong presence at conferences. It should be noted that a combination of factors matters for motivation to publish. One of the co-authors from Group B had a strong background in Group B's topic (and this person was not yet a full professor, which might be related to a greater motivation to publish.)

Conference organizing experience. Some Group A and B leaders mentioned that they had previous experience leading and/or running a conference (e.g., recruiting speakers). They said that they were fully aware and prepared for the extensive and challenging work required to run a new conference. In contrast, a Group C leader had limited familiarity organizing a conference, and this person appreciated learning from the experience. This respondent suggested he/she would do things differently in the future such as not wait to get information necessary to run her group. One Group C leader also mentioned that conference organizers did not provide certain materials an information that were supposed to be given during preparation for the session, which may have impacted the session.

Maintaining a shared vision. In many ways, conference organizers had a shared vision among themselves and with group leaders that facilitated accomplishment of conference goals. Conference organizers, however, did not always have consistent shared vision. They varied, reportedly, in how much they prioritized these goals: (a) generate collaboration between existing researchers for publication, (b) get educators to adopt researchbased instructional methods and collect data in their classes, and (c) bring new teacher-scholars together and get them interested in each other's ideas, which would expand the population of researchers who might support each other and work together.

One conference organizer felt that although several discussions were held before the conference about what the working groups should do, the leaders of Groups A, B, and C showed different skills in enacting the vision. The leaders in Groups A and B seemed to be able to enact the vision of collaborating on a manuscript, possibly partly because the group organizer who prioritized and valued collaborative writing the most was in one of those groups. (There were multiple goals for groups, and all group leaders were reported to have accomplished other goals well).

The leaders in Group C may not have had enough conversations ahead of time to help leaders understand, agree with, plan, and execute the plan to write publications, according to one respondent. The Group C leaders may not have agreed or were not skilled at enacting the vision (e.g., publication writing).

Conference organizers felt they were not always in agreement about exactly what their roles were. This would have been less challenging if the host university had provided more resources such as a staff member to help run the conference.

Oversight by central leadership. Conference organizers had difficulty figuring out how to balance their administrative duties--making the conference run smoothly and troubleshooting administrative problems-with their desire to be in the rooms attending presentations and helping to lead discussions all day.

Conference organizers felt that they were careful to work with group leaders and have planning meetings prior to the conference. Yet in retrospect, conference organizers realized that more oversight was needed before and during the conference. They said that at least one conference organizer should have gone to each group at the conference and ensured that the conference goal of publication writing was being achieved. Conference organizers stated that they wanted to participate in Group C, but only participated in Groups A and B. (Many individuals who missed attending Group $\mathrm{C}$ said that they wanted to attend the attractive-sounding topic of Group C). 
In Group A, attendees said the group had highly engaged, interactive, knowledgeable, and motivated participants, partly due to a strong voice of leadership in the room. It should be noted that multiple people chose this group as their top choice, so this group may have generally been a more popular topic for conference participants, which might explain why people were even more motivated to publish together. A strong presence was maintained by conference organizers in Groups A and B, and they helped make sure the groups made progress on their ideas and on planning a publication, according to interviewees.

Group $\mathrm{C}$ had no strong conference organizer presence. One conference organizer realized later it might have been helpful to have one of them in the room to help guide conversations. Group $\mathrm{C}$ had group leaders who tried to motivate group members. Group C members were not highly motivated, however. Group C did not find a champion or others to organize a paper.

One interviewee indicated that Group $\mathrm{C}$ had a few attendees in the room who would have been interested in leading the paper or writing sections of the paper, and some of these people were leaders in other ways during the conference day. These attendees were not sure what the topics or structure of the paper would be, and they were not sure who was leading the paper writing. The attendees felt like they were "just participants" and not the group leaders, so didn't want to "step on the toes of the Group C leaders in the room." Another interviewee indicated that participants and leaders were feeling a little shy or overly formal. This person suggested that it might be a helpful if the group attendees or leaders would have stepped in and helped the group make more progress.

One respondent in Group C commented on how leaders could have helped: "Group C was different than Group B. Group B did not spend a lot of time trying to decide who would be the leader or what the structure of the paper would be. Instead, Group B spent more time working on ideas for papers (including a summary of the themes generated in the group during the conference). Group members were to be co-authors writing about what they do in their classroom. One respondent remarked, "What a nice idea....write a quick paper about what I do in the classroom. If our group had heard that idea [in Group C], I think I would have been much more likely to write a paper for publication."

In other words, oversight as well as an easy-to-follow model of a publication idea would have been appreciated by group members. Respondents indicated that it would have been helpful if a conference organizer or leader had been in Group C and said, "It's easy to write a paper together. Here's a model paper we are using in Group B. It will take less than 2 hours of time commitment from authors. One author explains how this topic is taught in their own classroom. Other authors do the same. Then one person writes the introduction and conclusion. If that had happened, Group C might not have overestimated the time needed to write a paper for publication."

Rewards and motivation of non-full professors. The promotion and reward systems established by universities provides rewards for professors for publishing if they are not yet promoted to the rank of full professor. Unlike full professors (who have relatively minimal rewards for publishing), people who have the rank of postdocs, assistant professors, and associate professors usually need to publish as part of their jobs.

In the groups, it was not full professors who were champions of the papers. Non-full professors led writing efforts during and after the conferences, according to one respondent. When asked why, one respondent mused, "Assistant professors and associate professors are 'hungrier to publish."' Another respondent indicated that full professors are required to teach but are not required to do research, and thus, many full professors cease to publish research, especially when they have other commitments such as a higher service load in their departments. Another responded added, "Full professors can spend more time working on other projects such as service to the university and starting new centers. The main way to be promoted after you are full professor is to become an administrator. Leading university committees, initiatives, and centers are more important than publications for any full professors who want another promotion." 
A conference leader who enjoys collaborative discussions and who had recently been granted full professorship explained why he/she was not championing a paper nor co-authoring a collaborative manuscript at the conference. "I need to do my own work at this point. I'm tired of doing other people's work." This individual sounded a bit unmotivated and worn out from leadership responsibilities and collaborating with others while having other unfinished publications. Furthermore, this respondent added that his/her group's discussions ended up going in a particular direction that was not very interesting or relevant to this individual.

Small group size. One social scientist and interviewee felt that the group size might have affected how informal, empowered, and engaged group members felt. Perhaps if Group C had been smaller in size, group members might have felt more comfortable speaking up and not worried about "stepping on others' toes."

There have been some mixed results in studies of whether small groups perform better. In a study in which team sizes ranged from 5 to 12 members working on specific goals in a hospital, smaller teams were found to be more effective at meeting the assigned goals (Vinokur-Kaplan, 1995). Other studies indicate smaller teams show better performance (Gooding and Wagner, 1985; Ingham, Levinger, Graves, and Peckham, 1974; Pelled, Eisenhardt, and Xin, 1999). According to one meta-analysis, however, larger teams showed slightly better performance on complicated tasks in uncertain environments (Stewart, 2006).

Larger groups can have greater access to resources (e.g., time, money, and expertise), but smaller groups can have better communication and less social loafing. Overall, small groups are generally more likely to be more successful at quickly completing goals. Many other factors may play a role as well, including the function and characteristics of the group. Small and medium-sized research centers (up to 50 members) generated interdisciplinary knowledge more than large centers did (Rhoten, 2003). (Definition of small teams is not consistent across studies. 20-member teams are considered large in some studies.) When a group is medium or large in size, sometimes smaller sub groups form and work together more efficiently and have faster productivity as they make "middle-range" linkages or intellectual integration that is not shared across the entire group (Fuqua, 2002).

Conference organizers tried to keep the number of participants roughly equal when the conference began so that no group was particularly large. An observer walking by the classrooms said that Group A's group size appeared small, Group B's appeared medium, and Group C's appeared large. By the afternoon session, conference organizers agreed that Group A was small, Group B was a bit larger, and Group C was largest. One respondent in Group A felt that keeping Group A small was a good idea. This person implied that when group gets too large, interdisciplinary collaborative discussion is more difficult. This respondent had much experience organizing STEM-education conferences.

Physical environment: Meeting room and seating configuration. The physical environment may have also influenced how creative members felt, how easy it was to feel engaged rather than to sit back and feel isolated, and how easy it was to communicate and foster a shared vision of an intellectual collaborative interdisciplinary product. Group A had a working space which might have been ideal for fostering shared discussions during the afternoon. One interviewee commented that the room appeared great for attempting to develop a shared vision of outcomes for the group. A person in the group recalled that the room was a nice, rather small meeting room with one long table and seats that faced each other. The participant said,

"I liked the feel of the room. It encouraged participation. It was hard to 'hang back' in it. I could hear well. I could talk without having to drown out other conversations. My comments could be heard easily. Overall, it was easy for participants to see each other and feel part of one conversation."

Group B was held in a moderately sized room. In contrast to Group A and B, Group C was assigned a large room (e.g., a large classroom in the afternoon and a large lecture hall in the morning). The large classroom in the afternoon "appeared to be a regular classroom with tables that did not move around," recalled one respondent. "It felt too large." Seating was around small tables with some chairs facing away from the front and from others, making it difficult to generate a shared collaborative vision. It was not easy to have small group 
discussion, and the main leaders and presenters appeared relatively far away at the front of the room. One Group C respondent commented about the lack of a nice space:

"I didn't really know anyone near me in the room. If I am collaborating with new people and trying to be creative, I prefer to be in a nice, interesting space where I feel inspired and sociable- a space that allows me to more easily move my desk and change the physical environment so that I can collaborate...a space that feels more interesting can help me feel more engaged and creative than a regular classroom. At a teaching conference, I was in a fantastic, renovated large room. I felt engaged, inspired, focused, and maintained a positive attitude. I was able to talk well with others and listen to the leaders because the space made it easy to listen and communicate. In the past, I was part of an initiative that was a large, national interdisciplinary collaboration of PIs. The national funding agency scheduled meetings at unique retreat locations with the goal of enhancing collaboration. People talked about how great and inspiring those locations were. Couldn't the university support us being in one of the nicer rooms? In the future, I would enjoy coming to this conference again, but I would like to be in a nicer space that fuels my creativity." This individual mentioned that physical environmental research has indicated that creativity is enhanced by the physical environmental factors.

Process/Outcome: Positive evaluative responses. Having a positive attitude toward the working group might facilitate the outcome of publications.

More positive attitudes were held by Group A and B members than Group C members, according to the interviews and questionnaires completed at the end of working group session. Happiness with how the group went, engagement with the group, and optimism for future group collaboration were expressed more in Group A and B interviews than in Group C. Compared to Group C, Groups A and B provided higher ratings of the working group session, as shown below in Table B. A one-way ANOVA (as shown in the table below) indicated that the differences were statistically significant.

Table B.

Means and ANOVA results for Evaluations for Group A, B, and C after the afternoon Working Group

\begin{tabular}{|c|c|c|c|c|c|c|}
\hline Survey Item & Group & $\mathrm{N}$ & Mean & $\mathrm{SD}$ & $\mathrm{F}$ & Sig. \\
\hline \multirow[t]{3}{*}{ Content } & $\mathrm{A}$ & 8 & 4.75 & 0.46 & 7.508 & $0.003^{* *}$ \\
\hline & B & 12 & 4.75 & 0.45 & & \\
\hline & $\mathrm{C}$ & 7 & 3.857 & 0.69 & & \\
\hline \multirow[t]{3}{*}{ Presentation } & A & 8 & 4.63 & 0.52 & 6.968 & $0.005^{* *}$ \\
\hline & B & 9 & 4.89 & 0.33 & & \\
\hline & $\mathrm{C}$ & 6 & 3.67 & 1.03 & & \\
\hline \multirow[t]{3}{*}{ Opportunities for Engagement } & $\mathrm{A}$ & 8 & 5 & 0 & 2.976 & $0.06^{\mathrm{a}}$ \\
\hline & B & 12 & 5 & 0 & & \\
\hline & $\mathrm{C}$ & 8 & 4.75 & 0.46 & & \\
\hline \multirow[t]{3}{*}{ Interaction with others } & A & 8 & 5 & 0 & 5.357 & $0.01 * *$ \\
\hline & B & 12 & 5 & 0 & & \\
\hline & $\mathrm{C}$ & 8 & 4.63 & 0.52 & & \\
\hline \multirow[t]{3}{*}{ Interdisciplinary Exchange } & A & 7 & 4.57 & 0.79 & 1.253 & $\begin{array}{l}0.304 \\
\text { (not sig.) }\end{array}$ \\
\hline & B & 12 & 4.92 & 0.29 & & \\
\hline & $\mathrm{C}$ & 8 & 4.63 & 0.52 & & \\
\hline
\end{tabular}

Survey question: "On a scale from 1 [low] to 5 [high], please rate the overall session: content, presentation, opportunities for engagement, interaction with others, and interdisciplinary exchange."

${ }^{*} \mathrm{p}<=.05,{ }^{*} \mathrm{p}<=.01$, a $\mathrm{p}=.06$ statistical trend (two-tailed $\mathrm{t}$ test) 
Table C.

Perceptions of Group A, B, and C working group at the conference*

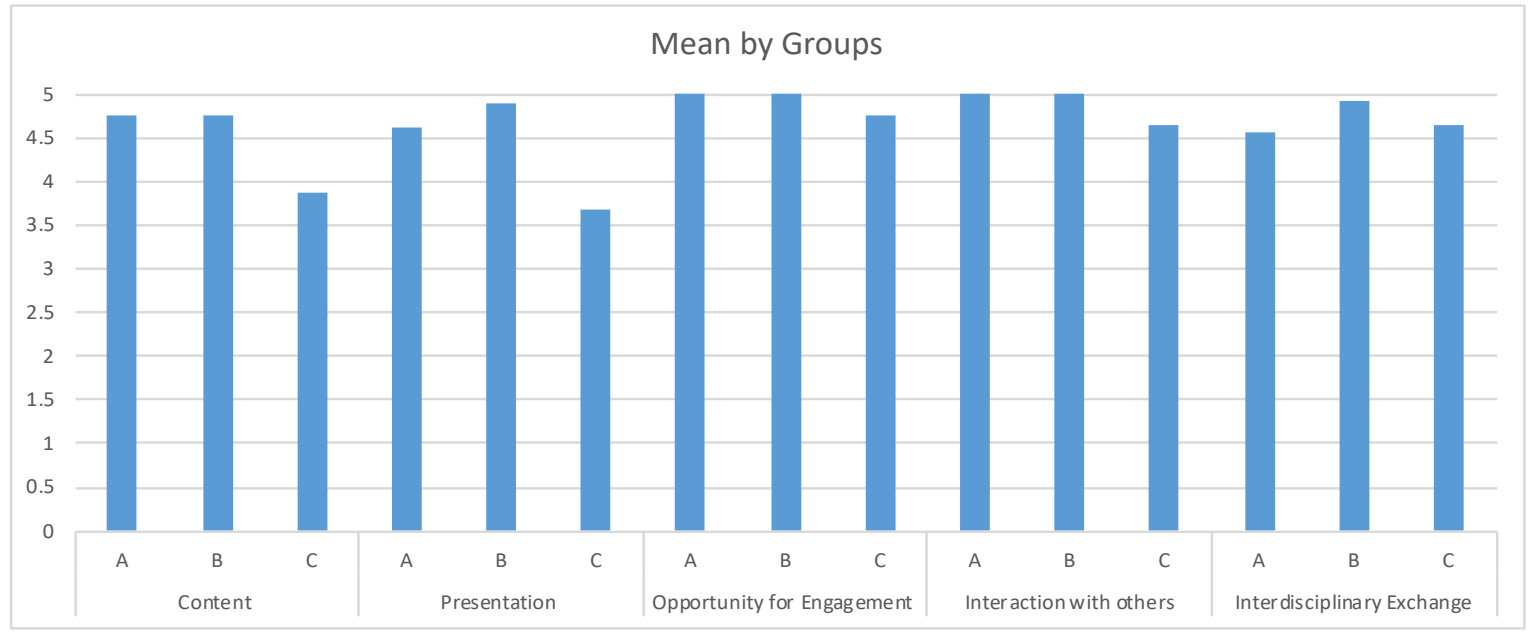

*This chart shows the Table B means in bar graph form.

One limitation is that rather few participants (approx. 25\%) completed the questionnaires, possibly because these working group questionnaires were handed out at end of the day before the conference dinner. Multiple individuals said they needed to leave early to reduce their exposure to heavy Los Angeles area traffic. It is unknown whether the results would have been different if more people had participated. One participant in group C (the largest group) did not complete the questionnaire because she did not want to provide negative ratings that might make anyone, such as hard-working leaders, look overly 'bad'. "I didn't want to fill out the questionnaire for Group C. The conference organizers and sessions leaders put a lot of time and effort into it. I appreciated their efforts. However, I was not totally satisfied or engaged in the working group." This individual did not seem upset, but merely had expectations that were not met.

\section{Outcomes}

Publication manuscripts. As of the writing of the present paper, two manuscripts have been submitted by Group A, which means their group was the most productive, defined in terms of completing publication drafts. Group B has shown some progress. They have one manuscript that is reportedly "well underway," another in progress, and a third possibly in progress. They have exchanged some emails and ideas in the past few months about the manuscript. One of the papers might be submitted by two group members who started a manuscript before the conference, and have revised it based on ideas from the conference. Group C has not made progress yet on writing or submitting a collaborative paper. (Again, there are many reasons why this group might be the least ready to collaborate.)

In case this report appears to be overly critical of Group B or Group C for being less ready to submit publication manuscripts, we would like to reiterate that writing manuscripts for publications was only one of several desired outcomes of the conference. Overall, the conference was highly appreciated and valued by many attendees in all groups. Teacher-scholars at the conference were expected to learn new scholarly work on three themes (problem-solving skills, computational skills, and equity), be inspired to gather more information about these themes, and be inspired to improve teaching practices, and find future potential collaborators who could provide support for future endeavors.

Analyses have not been conducted to determine whether Group A and B papers are transdisciplinary and collaborative. Perhaps the group most ready to collaborate (group A) will not be the group that accomplishes transdisciplinarity over time. 
A long-term collaboration. While the present paper is focused primarily on comparing the three groups' readiness to write publication manuscripts (esp. interdisciplinary collaborative scholarly manuscripts), there are many other ways to define the short- or long-term success of a conference (e.g., new ideas for teaching STEM, social support for ideas, new ideas for publication, long-term collaborations). Also, an individual attendee might gain ideas that lead to novel ideas in a solo-authored publication or a new teaching strategy which reduces the $\mathrm{D} / \mathrm{F} / \mathrm{W}$ rate ( $\mathrm{D}$ or F letter grades and withdrawals).

A time frame longer than a few months is needed to see whether ideas evolve, and what factors facilitate manuscript writing. One conference organizer said:

"I envisioned the paper publications as a carrot [incentive] or a short-term outcome, not the final outcome of a collaboration. I envisioned that people could continue a more long-term collaboration or at least a network...I don't know how well these paper publications are leading to a long-term collaboration. I want to know what other collaborations people have begun and what other outcomes people are achieving in the short-term and long-term."

An organizational psychologist explained that more time is needed to assess the outcomes of small group endeavors, and three years is a better marker of the outcomes of a group than three months.

\section{Concluding Thoughts}

More information about the antecedents, processes, and outcomes of interdisciplinary scientific collaborative efforts is needed. Further research and more passage of time such as a few years is needed to determine the quantity, quality, span of disciplinarity, novelty, and generativity of groups.

It is possible that groups with characteristics such as Group A may be smooth-running and productive over a short period of time. It is not clear whether such groups will continue being productive over a longer time frame. Furthermore, it is not clear whether the most heterogeneous groups will eventually develop the most novel ideas, that will lead to important innovations in STEM education.

Different definitions of success and ways of measuring success are needed. The number of manuscripts submitted to a journal within a few months after a conference is a limited way of measuring success. The quality, quantity, novelty, generativity, and many other factors should be assessed as well. Some innovations may impact education and society at large by ameliorating problems through programs and policies, but their effectiveness may not be realized for quite some time.

This paper focused on whether three working groups from a conference/collaboration published manuscripts, but the concepts could be applied to larger endeavors with different outcomes. The themes in the present paper are likely to generalize to other interdisciplinary scholarly collaborative endeavors. We recommend that teacher-scholars in any phase of a collaboration (planning to collaborate, collaborating, or reflecting on collaboration) consider the factors outlined in this paper as they consider the success of their team.

Our results suggest that several antecedent conditions and processes may impact group productivity. In particular, disciplinary span, leadership, physical environment, social cohesion, and group size may influence individuals' readiness to develop innovations such as interdisciplinary, collaborative publications. A narrow disciplinary span may lead groups to more rapid, smooth-running collaboration that results in innovative interdisciplinary collaborative products and publications. Products that emerge from collaborations with a wide disciplinary span might be more novel, generate more important new ideas, and have a bigger impact on science and society. Numerous other variables may influence collaborative productivity. More research is needed, particularly empirical research, to track and evaluate the processes and outcomes of interdisciplinary scientific and scholarly collaborative endeavors. 


\section{Acknowledgements}

We would like to thank the National Science Foundation for supporting the Breaking Boundaries conference/collaboration (Award \# 1644470). We would also like to thank the Breaking Boundaries leaders and participants for their insights.

\section{References}

Festinger, L., Schachter, S., \& Back, K. (1950). Social pressures in informal groups: A study of human factors in housing. Oxford, England: Harper.

Fuqua, J. (2002). Transdisciplinary scientific collaboration: An exploration of the research process (Unpublished doctoral dissertation). University of California, Irvine, CA.

Fuqua, J., Stokols, D., Gress, J., Harvey, R., \& Phillips, K. (2004). Transdisciplinary collaboration as a basis for enhancing the science and prevention of substance use and abuse. Substance Use and Misuse, 39, 14571514.

Gooding, R. Z., \& Wagner III, J. A. (1985). A meta-analytic review of the relationship between size and performance: The productivity and efficiency of organizations and their subunits. Administrative Science Quarterly, 462-481.

Hoogendoorn, S., Oosterbeek, H., \& van Praag, M. (2013). The impact of gender diversity on the performance of business teams: Evidence from a field experiment. Management Science, 59(7), 1514-1528.

Ingham, A. G., Levinger, G., Graves, J., \& Peckham, V. (1974). The Ringelmann effect: Studies of group size and group performance. Journal of Experimental Social Psychology, 10(4), 371-384.

Klein, J. T. (1996). Crossing Boundaries: Knowledge, Disciplinarities and Interdisciplinarities Knowledge: Disciplinarity \& Beyond. Indiana: Wayne State University Press

Langford, S. (In Press). Diversity in work teams. In B. J. Carducci (Editor-in-Chief) \& J. S. Mio \& R. E. Riggio (Vol. Eds.), Wiley-Blackwell Encyclopedia of Personality and Individual Differences: Vol. IV. Clinical, Applied, and Cross-Cultural Research. Hoboken, NJ: John Wiley \& Sons.

National Cancer Institute (1999). Transdisciplinary Tobacco Use Research Centers. Retrieved August 31, 2017, from https://cancercontrol.cancer.gov/brp/archive/tturc/index.html

National Research Council (2012). Discipline-Based Education Research: Understanding and Improving Learning in Undergraduate Science and Engineering. Washington, DC: The National Academies Press. https://doi.org/10.17226/13362. (This report was developed by the Division of Behavioral and Social Sciences and Education; Board on Science Education; Committee on the Status, Contributions, and Future Directions of Discipline-Based Education Research; Susan R. Singer, Natalie R. Nielsen, and Heidi A. Schweingruber, Editors.)

Pelled, L. H., Eisenhardt, K. M., \& Xin, K. R. (1999). Exploring the black box: An analysis of work group diversity, conflict and performance. Administrative Science Quarterly, 44(1), 1-28.

Rhoten, D. (2003) A Multi-Method Analysis of the Social and Technical Conditions for Interdisciplinary Collaboration (Final Report for National Science Foundation-funded project). September, 2003. Available at: http://www.hybridvigor.net/interdis/pubs/hv_pub_interdis-2003.09.29.pdf.

Vinokur-Kaplan, D. (1995). Treatment teams that work (and those that don't): An application of Hackman's group effectiveness model to interdisciplinary teams in psychiatric hospitals. The Journal of Applied Behavioral Science, 31(3), 303-327.

Stokols, D., Fuqua, J., Gress, J., Harvey, R., Phillips, K., Baezconde-Garbanati, L., Unger, J., Palmer, P., Clark, M., Colby, S., Morgan, G., \& Trochim, W. (2003). Evaluating transdisciplinary science. Nicotine \& Tobacco Research, 5(1), S21- S39.

Stokols, D., Harvey, R., Gress, J., Fuqua, J., \& Phillips, K. (2005). In vivo studies of transdisciplinary scientific 
collaboration: Lessons learned and implications for active living research. American Journal of Preventive Medicine, 28(2S2), 202- 213.

Stokols, D., Misra, S., Moser, R.P., Hall, K.L., \& Taylor, B.K. (2008). The ecology of team science: Understanding contextual Influences on transdisciplinary collaboration. American Journal of Preventive Medicine, 35(2S), 96-115.

Vodosek, M. (2007). Intragroup conflict as a mediator between cultural diversity and work group outcomes. International Journal of Conflict Management, 18(4), 345-375.

Woehr, D. J., Arciniega, L. M., \& Poling, T. L. (2013). Exploring the effects of value diversity on team effectiveness. Journal of Business and Psychology, 28(1), 107-121.

Zahn, G. L. (1991). Face-to-face communication in an office setting: The effects of position, proximity, and exposure. Communication Research, 6, 737-754. 